To appear in Journal of Fluids and Structures, 11 (4).

\title{
Sectional Lift Forces for an Oscillating Circular Cylinder in Smooth and Turbulent Flows
}

\author{
H. M. Blackburn* \\ W. H. Melbourne \\ Department of Mechanical Engineering, Monash University, \\ Clayton, Victoria 3168, Australia
}

\begin{abstract}
Measurements of sectional lift forces are presented that were obtained using a circular cylinder with forced cross flow oscillation in smooth and turbulent wind tunnel flows at a Reynolds number of $1.6 \times 10^{5}$. Maximum oscillation amplitudes were $3 \%$ of diameter. By employing variable-frequency forcing, the dimensionless oscillation frequency could be altered while the Reynolds number was held constant. With six transducers set out equi-spaced along the cylinder span it was possible to estimate spanwise correlation of lift based on simultaneous measurements, in addition to sectional lift coefficients.

Decomposition of lift forces into components correlated and uncorrelated with cylinder motion indicated that it was reasonable to assume that the uncorrelated component was unaffected by cylinder motion and that the observed increase in spanwise correlation of lift could be accounted for by the motion-correlated component. For smooth flow, examination of the magnitude and phase angle of lift force showed that lock-in was an intermittent occurrence at the amplitudes of motion employed. Motion-correlated forces were found to be less significant in turbulent flows, with changes in oscillation frequencies producing little observable effect.
\end{abstract}

\footnotetext{
${ }^{*}$ Current address: CSIRO, Division of Building, Construction and Engineering, PO Box 56, Highett,
} Victoria 3190, Australia. 


\section{Introduction}

The motivation for the study of forces exerted by cross flowing fluid on bluff structures stems largely from the engineering design problem of predicting amplitudes of motion in vortex-induced structural vibration. While observations of wake dynamics (Ongoren \& Rockwell 1988, Williamson \& Roshko 1988, Blackburn \& Henderson 1995) are an essential aid to fuller understanding of the fluid-structure coupling, measurement and description of fluid-induced loadings are central to the task of providing simplified engineering models of the fluid-structure interaction.

It has been apparent for some time that even at low amplitudes of cross flow motion, there can be measurable interaction between structural motion and wake dynamics. This is manifested in observations of motion-correlated sectional forces and an increase in the spanwise correlation of fluctuating forces. These are each significant, since increased spanwise correlation leads to increased average fluctuating force per unit length, while velocity-correlated forces have the character of an aerodynamic damping; since structural damping values are typically low, motion-correlated forces can have a large effect on structural motion levels. Some engineering models that are employed for response prediction at low motion amplitudes (see e.g. Blackburn \& Melbourne 1993) attempt to combine a description of motion-correlated forces with effects of spanwise correlation. For example, the model for prediction of cross flow motion amplitudes proposed by Vickery \& Basu (1983) attributes the increase in spanwise correlation to motion-correlated forces in such a way that only the dependence of sectional forces on amplitudes needs to be described.

In this paper we describe an experimental programme which had the purpose of investigating motion-correlated lift forces at low amplitudes of motion. The experimental model was a circular cylinder with an aspect ratio 4.5:1, with six lift force transducers of short axial length (0.1 diameter) set out equi-spaced along the span. The cylinder could be forced in cross flow oscillation at amplitudes of up to $\pm 3 \%$ of diameter and measurements of lift force could be collected simultaneously from all the transducers. The frequency of oscillation was adjustable so that the reduced velocity (alternatively, dimensionless frequency) could be varied independently of Reynolds number, which was maintained constant at $1.6 \times 10^{5}$ during these tests. Tests were carried out in nominally smooth flow (turbulence intensity $0.6 \%$ ) and in turbulent flows ranging up to $18 \%$ intensity; most emphasis is placed on the results for smooth flow.

\section{Equipment}

The cylindrical wind tunnel model had a circular cross section of $200 \mathrm{~mm}$ diameter $(D)$ and length of $900 \mathrm{~mm}$, and was installed between large fixed end plates in the $2 \mathrm{~m}$ high by $1 \mathrm{~m}$ wide working section of the Monash University $450 \mathrm{~kW}$ wind tunnel. The end plates were rectangular, $9 \mathrm{D}$ width cross flow, $7 \mathrm{D}$ length streamwise, with the cylinder axis located $2.5 \mathrm{D}$ from the leading edge.

Nominally smooth flow in the working section had a longitudinal turbulence intensity of $0.6 \%$ (averaged over the cylinder span), while with the addition of a turbulence screen upstream of the model, turbulence intensities of $4.2 \%, 9.6 \%$ and $18 \%$ could be achieved. Turbulence integral length scales were found to be approximately $0.5 \mathrm{D}$. The mean flow velocities measured along the cylinder centreline position were within $2 \%$ of the spanwiseaveraged values for all flow configurations. 
Additional details concerning the working section, turbulence intensities, and measurements of lift made with the cylinder held fixed in smooth and turbulent flows can be found in Blackburn \& Melbourne (1996).

\subsection{Cylinder model}

The cylinder contained six force transducers, spaced at intervals of $0.75 \mathrm{D}$ along the span, with the remainder of the cylinder being made up of seven 'blank' sections which maintained the cylindrical shape. All these parts were mounted on an inner aluminium tube of $100 \mathrm{~mm}$ diameter for structural support. A diagram of the model is shown in figure 1. The blank sections were of built-up construction using thin aircraft plywood, with an outer finish of glass fibre an epoxy paint, rubbed back to a smooth, glossy finish. Axial gaps of $0.5 \mathrm{~mm}$ between the various cylinder segments were sealed with $6 \mathrm{~mm}$ wide strips of $30 \mu \mathrm{m}$ thick polyurethane elastomer which were glued to the cylinder after assembly.

Measurements of the assembled cylinder showed that the maximum variation in radius at any section was $0.2 \mathrm{~mm}$, while the standard deviation of diameter was $0.12 \mathrm{~mm}$.

\subsection{Force transducers}

The force transducers were designed with the objective of measuring cross flow forces acting on short sections of the cylinder, thus giving a good approximation to the sectional forces. A diagram of a force transducer is shown in figure 2. Each transducer consisted of a short $(0.1 \mathrm{D}$ axial length) segment of the cylinder which was mounted from the inner structural tube on beam springs. Connection to the inner structural tube was provided by a clamp ring. The springs had semiconductor strain gauges bonded to them, which in turn were connected in a full strain bridge circuit, one per transducer. The outer surfaces of the cylinder had the same finish as the blank sections.

In order to reduce inertial forces experienced by the transducers while maintaining rigidity, transducer outer segments were constructed from carbon and glass fibre composites, foamed plastics and balsa wood. The completed segments had a mass of $16 \mathrm{gm}$ for a $20 \mathrm{~mm}$ long segment of a $200 \mathrm{~mm}$ diameter cylinder and the first resonance frequency on the beam springs was $400 \mathrm{~Hz}$. Static measurements showed that the transducers were approximately 150 times more sensitive to cross flow than streamwise forces.

The data reduction, to be described in $\S 3$, required the motion of the cylinder to be known at each transducer location and to this end an accelerometer was mounted within each transducer, as shown in figure 2 .

\subsection{Cylinder oscillation system}

The cylinder assembly was provided with an oscillation system which enabled it to be driven cross flow in sinusoidal oscillation at amplitudes up to $\pm 6 \mathrm{~mm}(3 \%)$ in a frequency range $10-60 \mathrm{~Hz}$. The cylinder, connected in a mounting yoke, formed the main mass of a resonant spring-mass system which was driven at its resonant frequency by an electromagnetic shaker with tuned input provided by a signal generator. The resonant frequency could be varied by altering the lengths and cross-sections of steel beam springs. The driving yoke and cylinder were braced against unwanted motions with music wire stays. A sketch showing the layout of the rig was presented in Blackburn \& Melbourne (1992). 
Inside the working section, the cylinder spanned $900 \mathrm{~mm}$ between two large hardboard end-plates that were fixed $50 \mathrm{~mm}$ from the main tunnel walls. To allow relative movement between the oscillating cylinder and the fixed end plates while blocking flows around the ends of the cylinder, the small clearance was sealed with synthetic fur muffs fixed to the cylinder. The inner structural tube pierced both the end plates and the wind tunnel walls, but air was prevented from flowing into or out of the working section through the resulting holes by plenum boxes that surrounded the protrusions through the tunnel walls.

\section{Data Collection and Analysis}

During collection of experimental data, signals from the six force transducers and accelerometers were collected simultaneously using a Perkin-Elmer 3210 minicomputer equipped with an analogue-to-digital conversion board and an external clock source (clock rate $200 \mathrm{~Hz}$ for all results discussed here, giving a Nyquist frequency of $100 \mathrm{~Hz}$ ). Prior to sampling, the signals were passed through analogue anti-aliasing filters to remove any frequency components above $100 \mathrm{~Hz}$. All subsequent processing was carried out digitally. The main techniques employed are outlined below; more detailed descriptions are available in Blackburn (1992).

\subsection{Cancellation of inertial forces}

The technique upon which all subsequent signal processing relied was cancellation of inertial component of force transducer signal. Since the force experienced by an accelerating transducer sensor is the sum of aerodynamic force and the force required to accelerate the mass of the outer segment of the transducer, the inertial component of the force must be estimated and subtracted in order that the aerodynamic component can be studied. Accurate correction for inertial effects is crucial, since the interaction between oscillation and force applied by the fluid is of major interest: poor correction can leave forces at the frequency of motion which will be incorrectly assigned an aerodynamic origin.

A time domain method was employed for the task of estimating transducer inertial forces. First, a finite impulse response function (IRF) was identified to describe the relationship between accelerometer and strain bridge signals recorded with the cylinder oscillating (at selected amplitude and frequency) in the absence of flow. The IRF was then convolved with the signal from the transducer accelerometer, recorded in the presence of flow, to produce an estimate of the inertial component of force transducer response.

Adaptive digital filter techniques were deemed suitable for computation of finite IRF weights, and the method of fast least squares (Bellanger 1987) was implemented. After some testing, filters with 32 weights were used: it was found that the method converged rapidly and was capable, in the absence of flow, of cancelling force transducer signal to within the accuracy available given the analogue-to-digital conversion quantum of $5 \mathrm{mV}$.

\subsection{Estimation of cylinder velocity}

As mentioned above, each transducer segment carried its own accelerometer: these were calibrated against a servo-accelerometer reference prior to installation in the cylinder. In order to obtain cylinder velocities at transducer locations, a procedure based on the DFT and term-by-term integration of Fourier series was implemented. It was found that this 
method produced very accurate integration of signals (to within a constant of integration) over long time periods. Leakage effects corrupted the calculations at the start and end of the recorded timeseries, but this problem was overcome by discarding those data prior to computation of the coefficients presented in $\S \S 4$ and 5 .

\subsection{Re-introduction of added mass forces}

Bodies forced to oscillate in still fluid experience forces exerted by the fluid: if the amplitude of motion is small enough that flow separation does not occur, these forces are dominated by two components.

The first component, known as added mass force, is linearly related to the acceleration of the body. Physically, it is the force required to accelerate a mass of fluid that is moved from one location to another by the passage of the body (Milne-Thompson 1960, $\S 9.21-$ 2 ). In potential flow, the added mass of an infinite circular cylinder equals the mass of fluid it displaces. Experimental results for finite circular cylinders with similar oscillation amplitudes and Reynolds numbers to those used here (Sarpkaya 1978, Lundgren, BrinkKjær, Sand \& Jacobsen 1979, Moe \& Verley 1980), show that the added mass is close to that predicted for potential flow.

The second component is viscous force due to boundary layer shear stresses, which for low motion amplitudes is linearly related to cylinder velocity. Batchelor (1967) presents a boundary layer method for the estimate of this viscous force, from which it was estimated that viscous forces were two orders of magnitude smaller than the added mass forces for the parameters of this experimental programme.

The synthesis of digital filters designed to cancel out the signals produced by the cylinder force transducers when the cylinder was oscillated in the absence of flow resulted in the cancellation, not only of the unwanted inertial force necessary to accelerate the outer segments of the transducers, but also the added mass and viscous forces produced by moving the cylinder through the fluid. In order to correctly characterize the forces exerted by the fluid on the cylinder in the presence of flow, the forces which would have been produced by oscillating the cylinder in the still fluid were computed from the cylinder acceleration and added to the force transducer signals after convolution with the digital cancellation filters. As noted above, the viscous forces were estimated to be very small in relation to the inertial forces, smaller in magnitude than the analogue-to-digital conversion quantum. Consequently, only added mass forces were computed and reintroduced.

\subsection{Motion-correlated and residual sectional lift}

When discussing motion-correlated forces, the implication is that the correlation is performed over many cycles of cylinder motion, or until statistically steady values are reached. There are a number of ways of estimating forces correlated with motion, which are all essentially equivalent; in the past, different experimenters have used the method which best suited the equipment at their disposal. If the cylinder motion is close to being simple harmonic, the motion-correlated forces can be described by forces correlated with velocity and acceleration, with velocity and displacement, or equivalently by a magnitude and phase with respect to one of the three motion variables.

Given a signal proportional to cylinder acceleration, say $\cos 2 \pi f t$, and velocity, say $\sin 2 \pi f t$, the formulæ for estimating coefficients of lift correlated with acceleration and 
velocity from a record of coefficient of lift $C_{l}(t)$ over some averaging period $T$ are

$$
\begin{aligned}
C_{l a} & =\int_{0}^{T} C_{l}(t) \cos 2 \pi f t \mathrm{~d} t / \int_{0}^{T} \cos ^{2} 2 \pi f t \mathrm{~d} t \\
C_{l v} & =\int_{0}^{T} C_{l}(t) \sin 2 \pi f t \mathrm{~d} t / \int_{0}^{T} \sin ^{2} 2 \pi f t \mathrm{~d} t,
\end{aligned}
$$

where $C_{l a}$ and $C_{l v}$ are respectively the acceleration- and velocity-correlated lift force coefficients. Jones, Cincotta \& Walker (1969) used an analogue technique based on this formulation to compute the magnitude of forces correlated with cylinder displacement and velocity.

An alternative procedure is to use cross-spectral estimates. If $S_{l a}(f)$ is the (one-sided) cross-spectrum between force and acceleration and $S_{a}(f)$ the autospectrum of acceleration,

$$
\begin{aligned}
& C_{l a}=\int_{0}^{\infty} \Re\left[S_{l a}(f)\right] \mathrm{d} f / \int_{0}^{\infty} S_{a}(f) \mathrm{d} f \\
& C_{l v}=\int_{0}^{\infty} \Im\left[S_{l a}(f)\right] \mathrm{d} f / \int_{0}^{\infty} S_{a}(f) \mathrm{d} f,
\end{aligned}
$$

where $\Re$ and $\Im$ denote real (in-phase) and imaginary (out-of-phase) components.

The equivalent discrete method corresponding to (1) and (2) above, given the timeseries $C_{l}(k), \ddot{y}(k)$ and $\dot{y}(k)$ of lift coefficient, acceleration and velocity, each $N$ records long, is to compute

$$
\begin{aligned}
& C_{l a}=\sum_{i=1}^{N} \ddot{y}(i) \cdot C_{l}(i) / \sum_{i=1}^{N} \ddot{y}^{2}(i) \\
& C_{l v}=\sum_{i=1}^{N} \dot{y}(i) \cdot C_{l}(i) / \sum_{i=1}^{N} \dot{y}^{2}(i) .
\end{aligned}
$$

This amounts to least-squares fitting a linear relationship between lift coefficient and acceleration on the one hand, and lift coefficient and velocity on the other.

Since the acceleration and velocity are orthogonal functions for simple harmonic motion, the lift coefficients $C_{l a}$ and $C_{l v}$ are uncorrelated. Likewise, the residual lift coefficient process $C_{l r}(t)$

$$
\begin{aligned}
C_{l r}(t) & =C_{l}(t)-C_{l a} \cos 2 \pi f t-C_{l v} \sin 2 \pi f t \\
& =C_{l}(t)-C_{l a}(t)-C_{l v}(t)
\end{aligned}
$$

is uncorrelated with both $C_{l a}(t)$ and with $C_{l v}(t)$. Accordingly, for a zero-mean lift force process, the variance of coefficient of lift can be expressed as the sum of the variances of the uncorrelated components

$$
C_{l}^{\prime 2}=C_{l a}^{\prime 2}+C_{l v}^{\prime 2}+C_{l r}^{\prime 2}
$$

where primed quantities represent RMS values and e.g. $C_{l a}^{\prime}=2^{-1 / 2} C_{l a}$ for simple harmonic motion. After performing the decomposition, it is possible to examine the statistics of the uncorrelated residual $C_{l r}$, as well as those associated with the total lift force, $C_{l}$. The discrete method was the one adopted in the present work; an example outcome of the decomposition process is illustrated in figure 3. Estimates have been made of the likely 
errors in the reported values of $C_{l a}$ and $C_{l v}$ based on the finite sample size, the sensitivity of the transducers, the RMS cancellation error of $5 \mathrm{mV}$ (see $\S 3.1$ ) and typical experimental values of dynamic pressure, giving an expected RMS error in values of motion-correlated lift coefficients of approximately 0.02 .

Spectra of total lift force showed peaks at the cylinder oscillation frequency in addition to those close to the fixed-cylinder Strouhal frequency, except when these were nearly coincident, in which cases only single spectral peaks could be resolved. The effect of the least-squares decomposition was to remove the spectral peaks at the cylinder oscillation frequencies, or to reduce the area of the peak in those cases when only a single peak could be resolved originally. This effect can be seen in figure 4 .

\section{Results: Smooth Flow}

We present here investigations of three aspects of lift on the cylinder oscillating in smooth flow: the partition of sectional lift into motion-correlated and uncorrelated components $(\S 4.1)$, the spanwise correlation of total, motion-correlated and residual lift (§4.2), and phenomena of entrainment or lock-in $(\S 4.3)$. Periods of cylinder oscillation are given in dimensionless form as reduced velocities $V_{r}=U / f D$, where $U$ is the free-stream speed, $f$ the frequency of oscillation and $D$ the cylinder diameter. Zero-to-peak cylinder cross flow oscillation amplitudes $y$ are given in the form $\alpha=y / D$. In many of the results which are presented, the amplitude of oscillation was held nominally constant over a set of runs in which the oscillation frequency (i.e. $V_{r}$ ) was varied. In these cases the value of $\alpha$ presented is the average over the runs of the spanwise-averaged values of amplitudes.

No correction for tunnel blockage (10\%) has been applied, in line with results presented in Blackburn \& Melbourne (1996) for the fixed cylinder. As reported there, at $R e=$ $1.6 \times 10^{5}$ and in smooth flow, the Strouhal number for vortex shedding from the fixed cylinder was $S t=0.20$. Consequently the 'critical' reduced velocity at which the frequency of cylinder oscillation matched the frequency of vortex shedding for the fixed cylinder, $V_{r \mathrm{c}}=5.0$.

All data sets analysed here were recorded with a sampling rate of $200 \mathrm{~Hz}$ and were 8192 samples $(40.96 \mathrm{sec})$ in length. For $S t=0.20$, this corresponds to approximately 500 vortex shedding periods.

In figures 6, 7, 10 and 12, thin solid lines are drawn as visual aids but none represent any particular functional relationship.

\subsection{Sectional lift force}

Sectional lift force coefficients presented in this section are the averages of RMS values measured at the four most central transducers on the cylinder, except as noted.

\subsubsection{Total and residual lift forces}

Sectional lift coefficients are shown as functions of reduced velocity and motion amplitude in figure 5. The dashed line in each diagram gives the value of $C_{l}^{\prime}$ measured with the cylinder held fixed, 0.76. Overall, the values of the coefficients of residual lift were approximately independent of reduced velocity and were similar to those found with the cylinder fixed. In Blackburn \& Melbourne (1996) it was found that $C_{l}^{\prime}$ fell with increasing 
Reynolds number over the range $1.16 \times 10^{5}<R e<2.43 \times 10^{5}$; there, at $R e=1.44 \times 10^{5}$, $C_{l}^{\prime}=0.81$. Standard deviations of coefficient of total lift reached maxima near $V_{r}=V_{r \mathrm{c}}$.

The variation in values of $C_{l r}^{\prime}$ seen in figure 5 may partly be due to small changes in Reynolds number between one test and the next. While the nominal Reynolds number for this series of tests was $1.6 \times 10^{5}$, it actually varied in the range $1.54-1.65 \times 10^{5}$ from run to run, owing to a lack of repeatability in tunnel speed control. Since, as noted above, values of $C_{l}^{\prime}$ for the fixed cylinder decrease with increasing Reynolds number in this regime, the variation in values can be partly attributed to Reynolds number variations.

Figure 5 indicates that the difference between total and residual lift tended to increase near $V_{r}=V_{r c}$. In order to examine this trend in more detail, while reducing the effect of variability discussed above, the differences between total and residual lift coefficients were computed: these differential coefficients $C_{l c}$ are given by

$$
C_{l c}^{\prime}=\sqrt{C_{l}^{\prime 2}-C_{l r}^{\prime 2}}
$$

and reflect the magnitude of lift forces which were correlated with cylinder motion. It can be seen in figure 6 that reduced velocity and cylinder amplitude had a definite effect on motion-correlated forces, which were substantial, of the same order as total lift forces, near $V_{r}=V_{r \mathrm{c}}$.

\subsubsection{Motion-correlated lift force components}

Motion-correlated lift force component coefficients $C_{l a}^{\prime}$ and $C_{l v}^{\prime}$ are shown in figure 7 . It can be seen (as expected from fig. 6) that the amplitudes increased with $\alpha$ and were maximum around $V_{r}=V_{r \mathrm{c}}$. Velocity-correlated lift coefficients $C_{l v}$ were positive for $V_{r} \geq V_{r \mathrm{c}}$ : correspondingly in those cases there were long-time average mechanical power flows from the fluid to the oscillating cylinder (equivalent to negative aerodynamic damping). In most cases, values of $C_{l a}$ were negative (equivalent to positive added mass).

The evidence presented in figures 6 and 7 suggests that near $V_{r}=V_{r \mathrm{c}}$ for $\alpha=2.7 \%$, the magnitude of motion-correlated lift remained nearly constant but the average phase with respect to cylinder motion varied with $V_{r}$. In order to illustrate this more clearly the acceleration- and velocity-correlated components of lift $\left(C_{l a}^{\prime} \& C_{l v}^{\prime}\right)$ are plotted against one another in figure 8. It can be seen that near $V_{r}=V_{r c}$, the magnitude of motion-correlated force could be considered approximately constant.

In order to evaluate the degree of spanwise correlation of motion-correlated lift, it is possible to compare average values of coefficients of motion-correlated lift obtained in two ways. In the first method, the spanwise-mean of the sectional values can be obtained, while in the second, lift force and velocity timeseries can be spanwise-averaged prior to estimating motion-correlated lift coefficients. If the motion-correlated forces were well correlated along the cylinder span at each instant, then the ratios of the coefficients obtained by the two methods should be near unity. It can be seen in figure 9 that the ratio was close to unity on average. For comparison, a similar set of ratios for the residual lift $C_{l r}$ is also presented, and it can be seen that the ratios were then lower, closer to 0.85, reflecting the lower spanwise correlation of residual lift forces. For the results in figure 9, averages were taken over all six transducers, and the nominal amplitude of oscillation $\alpha$ was $2.7 \%$. 


\subsection{Spanwise correlation of lift}

Cross-correlations of sectional total and residual lift forces obtained at different locations along the span were computed, enabling estimates of spanwise correlation lengths to be made. The methods used to compute cross-correlation coefficients $\rho(\lambda)$, where $\lambda=$ $\left|z_{1}-z_{2}\right| / D$ is the dimensionless spanwise spacing, from $6 \times 6$ matrices of values

$$
\rho_{z_{1} z_{2}}=\left(\overline{C_{l_{1}} C_{l_{2}}}-\overline{C_{l_{1}}} \overline{C_{l_{2}}}\right) / C_{l_{1}}^{\prime} C_{l_{2}}^{\prime}
$$

and, subsequently, the dimensionless correlation length

$$
\Lambda=\int_{0}^{\infty} \rho(\lambda) \mathrm{d} \lambda
$$

were discussed in Blackburn \& Melbourne (1996).

The effect of cylinder motion was generally to increase the degree of spanwise correlation of lift, with the effect being most pronounced near $V_{r}=V_{r c}$. A set of example results for $V_{r}=V_{r \mathrm{c}}$ is presented in figure 10, where the correlation coefficients with the cylinder in motion can be compared with those found with the cylinder held fixed. The spanwise correlations of residual force (also shown) remained similar to those for the fixed cylinder.

Estimated dimensionless correlation lengths $\Lambda$ for total and residual lift forces at $\alpha=$ $2.7 \%$ are shown in figure 11 . The data show a fair amount of scatter, and it should be said that even for the fixed cylinder and with the Reynolds number nominally fixed, estimates of correlation lengths could vary by as much as $0.5 \mathrm{D}$ from run to run. In order to emphasize the effect of $V_{r}$ on $\Lambda$, the differences between correlation lengths for total and residual forces are shown in figure 12 .

It should be noted that if motion-correlated forces are perfectly correlated along the span, then, formally, $\Lambda$ becomes infinite. The 'correlation lengths' of total force in figures 11 and 12 only give an indication of the scale of increase in correlation provided by motion of the cylinder.

\subsection{Lift forces near $V_{r c}$}

A point of interest in the investigations was to examine the relationship between lift forces and cylinder motion, particularly near $V_{r}=V_{r \mathrm{c}}$, and at the higher amplitudes of motion employed. Examination of lift force spectra showed that they were singlepeaked for the range of reduced velocities which gave rise to the plateau in figure 6 for $\alpha=2.7 \%$, as can be seen in figure 4. Initially, spectra were computed using the method of overlapping segment-averaged periodograms (30 averages at 257 frequencies). A more detailed examination using maximum-entropy spectral estimation (200 poles) gave approximately the same spectral shapes: in all cases which fell within the plateau of figure 6 , only a single spectral peak could be resolved, with the location of the peak matching the cylinder oscillation frequency. This frequency-locking is often considered sufficient to describe the condition of entrainment, or lock-in, however the fact that the major part of the lift force process was uncorrelated with cylinder motion gave rise to doubts concerning spectral resolution, leading to the following time-domain study.

In order to examine the lift force magnitude, and its phase angle with respect to cylinder velocity, the magnitude and phase of lift and cylinder velocity were computed by taking the Hilbert transform of these two signals (Oppenheim \& Schafer 1975, Schumm, Berger \& Monkewitz 1994). By considering the difference between their phases we can 
examine the lock-in process in the time domain, since full entrainment is accompanied by the phase-locking of one process to another. The results of this processing are shown in figure 13, and are for a single transducer near the centre of the span.

It can be concluded from examination of figure 13(c) that complete entrainment was not achieved, since while there were extended periods over which the lift force maintained an approximately constant phase angle with respect to cylinder velocity, there were also times when the lift phase unlocked from cylinder motion and increased with time, until settling to approximately the same phase angle (modulo $2 \pi$ ) as before. These features were common to all the transducers, and for all values of $V_{r}$ examined, although the direction of phase traverse during unlocking events varied: while for $V_{r}>V_{r c}$ phase angles on average increased with time, they decreased with time when $V_{r}<V_{r c}$, which is consistent with the differences between periods of imposed cylinder motion and vortex shedding for the fixed cylinder.

\section{Results: Turbulent Flows}

The effects of the introduction of turbulence to the flow past the fixed cylinder will first be briefly reviewed. More extensive descriptions of measurements of sectional lift forces obtained with the cylinder held fixed in turbulent flows are given in Blackburn \& Melbourne (1996). In those results, the Reynolds numbers employed ranged from $1 \times 10^{5}$ to $5 \times 10^{5}$, while the results presented here are for $R e=1.6 \times 10^{5}$.

\subsection{Effects of turbulence}

Turbulence (longitudinal) intensities $I_{u}=u^{\prime} / U$ of $4.2 \%, 9.6 \%$ and $18 \%$ were employed. At $R e=1.6 \times 10^{5}$, the RMS sectional coefficients of lift for the fixed cylinder were approximately $0.18,0.21$ and 0.38 respectively.

The primary effect of the $4.2 \%$ intensity turbulence with the cylinder fixed was to induce the critical transition at lower Reynolds number than was observed in smooth flow (where $R e_{\text {crit }} \simeq 2.4 \times 10^{5}$ ). In this turbulent flow, at $R e=1.6 \times 10^{5}$ the spectral densities of lift forces showed no distinct spectral peaks (which would indicate organized vortex shedding), instead decreasing monotonically from low frequencies. Correlation lengths of lift were approximately $1 D(\Lambda \simeq 1)$, decreased from approximately $3.75 D$ in smooth subcritical flow (Blackburn \& Melbourne 1996).

For turbulence of $9.6 \%$ intensity, the lift force spectra for the fixed cylinder were again broad with no distinct peaks, however instead of monotonic decline of spectral density with frequency, the spectral shapes exhibited broad plateaux, extending out to reduced frequencies $f_{r}=f D / U \approx 0.35$. Again, $\Lambda \simeq 1$.

At $18 \%$ intensity, lift spectra for the fixed cylinder exhibited distinct but very broad peaks at $f_{r} \simeq 0.23$. Accompanying this change, correlation lengths of lift increased to approximately $1.5 \mathrm{D}$. Taken together, these two features are thought to indicate establishment of (broad-band) vortex shedding behaviour.

\subsection{Sectional motion-correlated lift forces}

Measurements of motion-correlated lift forces obtained with turbulent flows are presented in figure 14. The nominal amplitude of motion employed for these tests was $3 \% \mathrm{D}$. As for 
the results in $\S 4.1$, the values are the averages of the coefficients obtained at the four most central transducers on the cylinder. In each of the flows, the spectra of lift forces were very similar to those obtained with the cylinder fixed, with the exception of the presence of sharp peaks at the cylinder oscillation frequencies. In addition to the background spectra retaining similarity in shape to those for the fixed cylinder at the same Reynolds numbers, the magnitudes of coefficients of residual lift were also very similar to the coefficients of lift for the fixed cylinder.

In all three turbulent flows, the magnitudes of motion-correlated lift forces were much smaller than for smooth subcritical flow (especially near $V_{r}=V_{r c}$ ), and the velocitycorrelated forces were effectively zero.

\section{Discussion}

Results presented in Blackburn \& Melbourne (1996) indicate that with the cylinder held fixed in smooth subcritical flows, sectional lift forces measured using the experimental rig are likely to be larger (by a factor of 1.5 to 2) than those appropriate to an isolated, high aspect ratio cylinder, as a consequence of tunnel blockage and aspect ratio effects. This finding is also likely to be applicable to the magnitudes of the lift forces reported here. The qualitative effects of imposed cylinder oscillation on the vortex-shedding process and on lift forces may however be similar to those for a long, isolated cylinder. The effects of aspect ratio and blockage are likely to have been less important in the turbulent flows.

\subsection{Smooth Flow}

\subsubsection{Motion-correlated lift}

A number of experimental studies of motion-correlated lift forces have been published (e.g. Bishop \& Hassan 1964, Protos, Goldschmidt \& Toebes 1968, Försching 1968, Jones et al. 1969, Nakamura, Kaku \& Mizota 1971, Yano \& Takahara 1971, Sarpkaya 1978, Middlin \& Simmons 1983) which have in the main concentrated on forces correlated with cylinder velocity since these are often the most important in the context of vortex-induced cross flow vibration. In these studies, motion-correlated force coefficients have been nondimensionalized in a variety of ways, but when reduced to a common basis they all show behaviour similar to that seen in figure 7: i.e. $C_{l v}>0$ when $V_{r}>V_{r \mathrm{c}}, C_{l v}<0$ when $V_{r}<V_{r \mathrm{c}}$.

The change of phase of motion-correlated forces with variation of $V_{r}$ is consistent with previous flow visualization results, e.g. those of Ongoren \& Rockwell (1988) which show a rapid change of phase of vortex shedding as reduced velocity is changed near $V_{r}=V_{r c}$.

In many of the previous experimental investigations of motion-correlated forces, scant attention has been given to the magnitude of the uncorrelated residual lift forces. The results presented here indicated that at low motion amplitudes the residual lift was similar to the lift experienced by the fixed cylinder, both as sectional values and in terms of correlation along the span. Also significant is the fact that the motion-correlated and residual forces were of similar order near $V_{r}=V_{r c}$, even for the low amplitudes of motion employed. 


\subsubsection{Entrainment}

The results presented in $\S 4.3$ indicate that entrainment, or lock-in of lift forces to cylinder motion, was an intermittent occurrence: this is in accord with the finding that the majority of lift force was uncorrelated with cylinder motion in all of the tests. It is possible that the intermittency was partly caused by significant background levels of flow turbulence $\left(u^{\prime} / U=0.6 \%\right)$, and would be reduced in smoother flows. One would also expect intermittency to reduce with increasing motion amplitudes.

At much lower Reynolds numbers $(100<R e<300)$ Koopmann $(1967)$ reported a $0.1 D$ threshold oscillation amplitude for lock-in to occur, and all the amplitudes employed here were well below this limit. The method used by Koopmann to assess lock-in, that requires a steady limit cycle to be observed when a cylinder motion variable is plotted against a fluid motion variable, is probably the most reliable available, and can indicate sub- and super-harmonic lock-in in addition to the primary lock-in investigated here. As indicated in $\S 4.3$, it is not necessarily a simple matter to decide if full lock-in has occurred on the basis of spectral measurements alone.

\subsection{Turbulent Flows}

It is apparent from a comparison of figures $7(c)$ and 14 that at the same amplitude of motion (nominally $3 \% D$ ), motion-correlated forces were much smaller in magnitude for all the turbulent flows than for smooth flow. Also the motion-correlated forces were dominated by acceleration-correlated components, with velocity-correlated components being effectively zero in all cases.

The small sizes of motion-correlated forces in these turbulent flows, where any vortex shedding present was poorly organized in time and space, indicates that both organized vortex shedding and cylinder motion at a frequency commensurate with it are prerequisites for substantial motion-correlated forces. For example, it is interesting that while in Blackburn \& Melbourne (1996) broad spectral peaks centred at $S t=0.23$ were noted in the lift force spectra for $I_{u}=18 \%$, there does not seem to be any corresponding interaction with cylinder motion near $V_{r}=1 / 0.23=4.4$ visible in figure $14(c)$. It may however be possible for large-amplitude motions to re-establish vortex shedding in some flows where it has broken down, for example as a result of the critical Reynolds transition for flows past circular cylinders: this phenomenon was indicated by some of the results presented by Wooton $(1969$, ฯ 29$)$.

In general, it is certainly possible for motion-correlated forces to exist in turbulent flows past circular cylinders, which conclusion can be drawn on the basis that large-amplitude oscillations of slender flexible structures are often observed in applications where the free stream is turbulent to some degree. In these situations not only the Reynolds number and turbulence intensity and but also the ratio of the integral length scale to the cylinder diameter (alternatively the ratio of eddy turnover time to vortex shedding period) are important determinants of behaviour. To draw stronger conclusions in this area it will be necessary to conduct experiments in situations where the base flow is comparatively insensitive to Reynolds number and perturbation effects, e.g. either by employing sharpedged sections or by choosing Reynolds numbers judiciously. 


\section{Conclusions}

At the amplitudes of motion examined, lift forces were incompletely correlated with cylinder motion. Statistics of the residual, uncorrelated, lift were similar to those for the lift on the fixed cylinder.

In smooth flow, the variation of motion-correlated sectional lift forces with reduced velocity was similar to that observed in other experiments. Motion-correlated forces were well correlated in the spanwise direction. Near $V_{r}=V_{r c}$, and for $\alpha \simeq 3 \%$, there was a plateau over which motion-correlated force magnitudes remained approximately constant, and phase angle with respect to cylinder motion changed, as $V_{r}$ was varied.

At the comparatively small motion amplitudes employed, time-domain studies of lift phase angle in smooth flow indicated that lock-in was intermittent, despite spectral features which indicated entrainment. The lift force process revealed by the present experiments is quite complex and not easily characterized, since it is neither completely random nor deterministic.

In the turbulent flows and at the Reynolds numbers employed here, motion-correlated forces were much smaller than were observed in smooth flow. Further work is needed to gain a better understanding of motion-correlated forces in turbulent flows, particularly those typically encountered in full scale, where the ratio of turbulence integral scale to cylinder diameter is often much larger than the value of 0.5 employed in the present experiments.

\section{Acknowledgements}

We would like to acknowledge financial support from the Australian Research Council through the course of this research. The first author would also like to acknowledge financial support provided in the early stages of this work in the form of a Monash Graduate Scholarship.

\section{References}

Batchelor, G. K. (1967). An Introduction to Fluid Dynamics, Cambridge University Press, London.

Bellanger, M. (1987). Adaptive Digital Filters and Signal Analysis, Marcel Dekker, New York.

Bishop, R. E. D. \& Hassan, A. Y. (1964). The lift and drag forces on a circular cylinder oscillating in a flowing fluid, Philosophical Transactions of the Royal Society of London A 277: 51-75.

Blackburn, H. M. (1992). Lift on an Oscillating Cylinder in Smooth and Turbulent Flows, $\mathrm{PhD}$ thesis, Department of Mechanical Engineering, Monash University.

Blackburn, H. M. \& Henderson, R. D. (1995). Near-wake vorticity dynamics in bluff body flows, 12th Australasian Fluid Mechanics Conference, Sydney, pp. 17-20.

Blackburn, H. M. \& Melbourne, W. H. (1992). Lift on an oscillating cylinder in smooth and turbulent flow, Journal of Wind Engineering and Industrial Aerodynamics 41: 79-90. 
Blackburn, H. M. \& Melbourne, W. H. (1993). Cross flow response prediction of slender circular-cylindrical structures: Prediction models and recent experimental results, Journal of Wind Engineering and Industrial Aerodynamics 49: 167-176.

Blackburn, H. M. \& Melbourne, W. H. (1996). The effect of free-stream turbulence on sectional lift forces on a circular cylinder, Journal of Fluid Mechanics 306: 267-292.

Försching, H. (1968). Aeroelastic instability investigations on prismatic beams, Symposium on Wind Effects on Buildings and Structures, Loughborough University, pp. 22.1-5.

Jones, Jr., G. W., Cincotta, J. J. \& Walker, R. W. (1969). Aerodynamic forces on a stationary and oscillating circular cylinder at high Reynolds numbers, Technical Report $R$-300, NASA.

Koopmann, G. H. (1967). The vortex wakes of vibrating cylinders at low Reynolds numbers, Journal of Fluid Mechanics 28(3): 501-512.

Lundgren, H., Brink-Kjær, O., Sand, S. E. \& Jacobsen, V. (1979). Improved physical basis of wave forces, Special Conference on Civil Engineering in the Oceans, Vol. IV, San Francisco, pp. 11-15.

Middlin, A. J. \& Simmons, J. M. (1983). Some effects of turbulence on power transfer to an oscillating cylinder in a cross flow, Journal of Wind Engineering and Industrial Aerodynamics 14: 267-278.

Milne-Thompson, L. M. (1960). Theoretical Hydrodynamics, 4th edn, Macmillan, London.

Moe, G. \& Verley, R. P. C. (1980). Hydrodynamic damping of offshore structures in waves and currents, Offshore Technology Conference, Houston. Paper 3798.

Nakamura, Y., Kaku, S. \& Mizota, T. (1971). Effect of mass ratio on the vortex excitation of circular cylinders, 3rd International Conference on Wind Effects on Buildings and Structures, Tokyo, pp. 727-736.

Ongoren, A. \& Rockwell, D. (1988). Flow structure from an oscillating cylinder. Part 1., Journal of Fluid Mechanics 191: 197-223.

Oppenheim, A. V. \& Schafer, R. W. (1975). Digital Signal Processing, Prentice-Hall, Englewood Cliffs, N.J.

Protos, A., Goldschmidt, V. W. \& Toebes, G. (1968). Hydroelastic forces on bluff cylinders, Symposium on Unsteady Flows, ASME, Philadelphia. Paper No. 68-FE-12.

Sarpkaya, T. (1978). Fluid forces on oscillating cylinders, ASCE Journal of the Waterways, Ports, Coastal and Ocean Division 104(WW4): 275-290.

Schumm, M., Berger, E. \& Monkewitz, P. A. (1994). Self-excited oscillations in the wake of two-dimensional bluff bodies and their control, Journal of Fluid Mechanics 271: $17-53$.

Vickery, B. J. \& Basu, R. I. (1983). Across-wind vibration of structures of circular cross section. Part 1, Journal of Wind Engineering and Industrial Aerodynamics 12: 49-73. 
Williamson, C. H. K. \& Roshko, A. (1988). Vortex formation in the wake of an oscillating cylinder, Journal of Fluids and Structures 2: 355-381.

Wooton, L. R. (1969). The oscillations of large circular stacks in wind, Journal of the Institution of Civil Engineers 43: 573-599.

Yano, T. \& Takahara, S. (1971). Study on unsteady aerodynamic forces acting on an oscillating cylinder, 3rd International Conference on Wind Effects on Buildings and Structures, Tokyo, pp. 737-746. 


\section{List of Figures}

1 Diagram of cylinder assembly, showing locations and dimensions of the six force transducers. An internal structural cylinder is responsible for the reduced-diameter protrusions at cylinder ends. All unspecified dimensions are in mm (upper), diameters (lower) . . . . . . . . . . . . . . . . . 17

2 Force transducer construction. . . . . . . . . . . . . . . . . . 17

3 Example showing decomposition of lift force into parts correlated with cylinder acceleration and velocity, with uncorrelated residual. ......, $C_{l}$; $C_{l r} ;-, C_{l a} ;--, C_{l v} \ldots \ldots \ldots \ldots \ldots$

$4 \quad$ Variation with $V_{r}$ of lift force spectra for a nominal oscillation amplitude of $2.7 \% D$ in smooth flow: (a): total lift force, $(b)$ : residual lift force. . . .

5 RMS values of total and residual lift in smooth flow. Lower dashed line in each graph indicates value of $C_{l}^{\prime}$ with cylinder held fixed at beginning of tests (0.76). Nominal oscillation amplitudes: (a), $\alpha=0.98 \% ;(b), \alpha=$ $1.9 \% ;(c), \alpha=2.7 \%$. $\square, C_{l}^{\prime} ; \triangle, C_{l r}^{\prime} ; \cdots \ldots, C_{l}^{\prime}$ (fixed cylinder). . . . . . . . .

$6 \quad$ Values of the differential (motion-correlated) coefficients of lift $C_{l c}^{\prime}$ (eq. 10) in smooth flow as functions of reduced velocity and amplitude. $0, \alpha=$ $0.98 \% ; \square, \alpha=1.9 \% ; \triangle, \alpha=2.7 \%$. . . . . . . . . . .

7 Coefficients of fluctuating lift correlated to cylinder acceleration $\left(C_{l a}^{\prime}\right)$ and velocity $\left(C_{l v}^{\prime}\right)$ in smooth flow as functions of $V_{r}$ at amplitudes of motion: (a): $\alpha=0.98 \%,(b): \alpha=1.9 \%,(c): \alpha=2.7 \% . \square, C_{l a}^{\prime} ; \triangle, C_{l v}^{\prime}$.

8 Coefficients of acceleration- and velocity-correlated lift $\left(C_{l a}^{\prime} \& C_{l v}^{\prime}\right)$ for $\alpha=$ $2.7 \%$ in smooth flow, with $V_{r}$ as a parameter. . . . . . . . . .

9 Comparison of ratio of value of $C_{l v}^{\prime}$ obtained from the spanwise-mean lift and cylinder velocity to the average value obtained from the six individual measurements to a similar ratio for the residual lift $C_{l r}^{\prime} . \alpha=2.7 \%$, smooth flow. $\circ, C_{l v}^{\prime} ; \square, C_{l r}^{\prime} . \ldots \ldots \ldots \ldots \ldots$

10 Effect of cylinder oscillation amplitude on spanwise correlation at $V_{r}=V_{r c}$ in smooth flow; (a): fixed cylinder, $(b): \alpha=0.98 \%,(c): \alpha=1.9 \%,(d)$ : $\alpha=2.7 \%$. $\circ$, total; $\triangle$, residual.

11 Spanwise correlation lengths $\Lambda$ for total and residual lift forces in smooth flow shown as functions of $V_{r}(\alpha=2.7 \%) . \triangle$, total; $\circ$, residual. . . . . . . 24

12 Difference between correlation lengths of total and residual lift forces as a function of $V_{r}(\alpha=2.7 \%)$ for smooth flow. . . . . . . . . . . . . . 25

13 Lift coefficient (a), magnitude envelope $(b)$ and $\phi$, phase relative to cylinder velocity $(c)$ at $V_{r}=5.14, \alpha=2.7 \%$ in smooth flow. Phase angles are given as multiples of $\pi \ldots \ldots \ldots \ldots \ldots \ldots$

14 Coefficients of fluctuating lift correlated to cylinder acceleration $\left(C_{l a}^{\prime}\right)$ and velocity $\left(C_{l v}^{\prime}\right)$ as functions of $V_{r}$ for nominal motion amplitude of $3 \% \mathrm{D}$ in turbulent flows. Turbulence intensities; (a): $I_{u}=4.2 \%,(b): I_{u}=9.6 \%$, $(c): I_{u}=18 \% . \square, C_{l a}^{\prime} ; \triangle, C_{l v}^{\prime}$. 


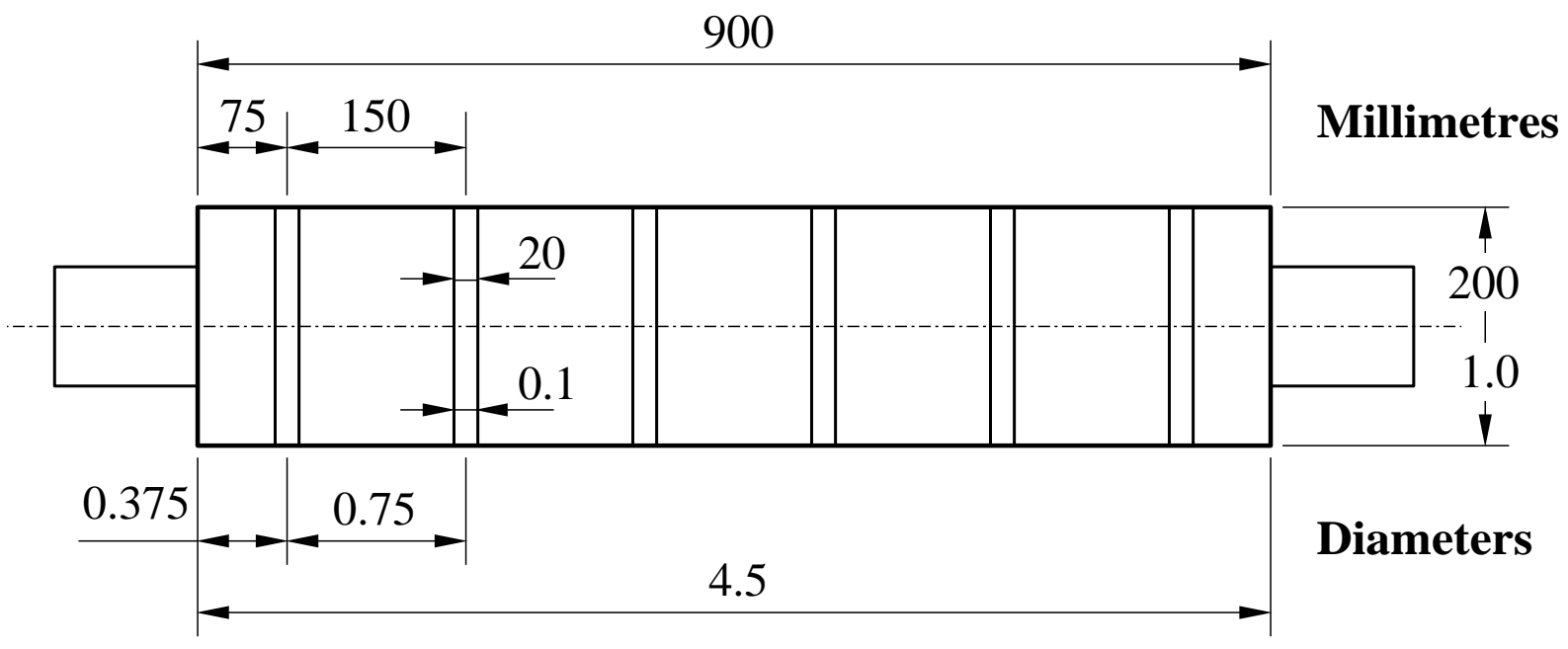

Figure 1: Diagram of cylinder assembly, showing locations and dimensions of the six force transducers. An internal structural cylinder is responsible for the reduced-diameter protrusions at cylinder ends. All unspecified dimensions are in mm (upper), diameters (lower).

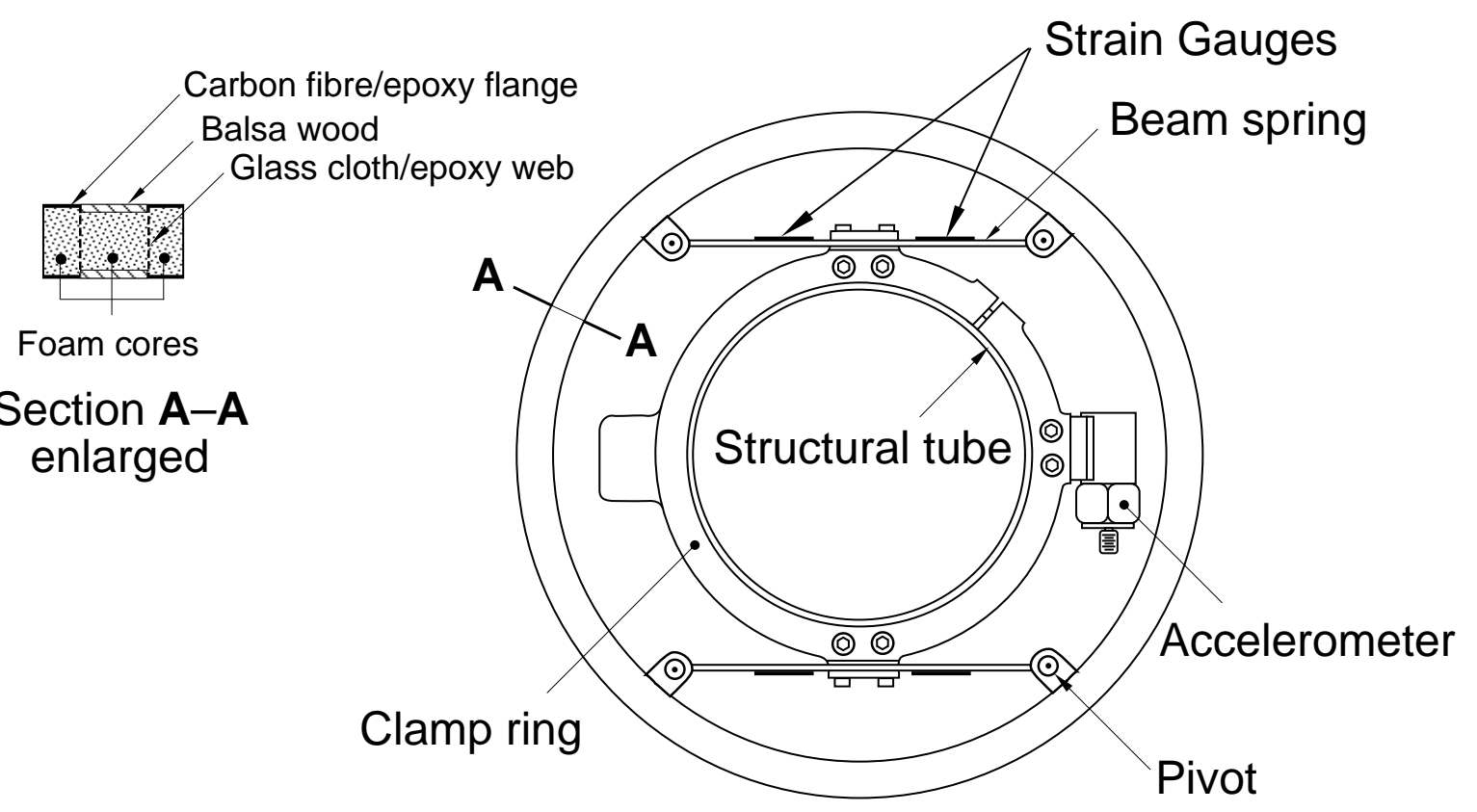

Figure 2: Force transducer construction. 


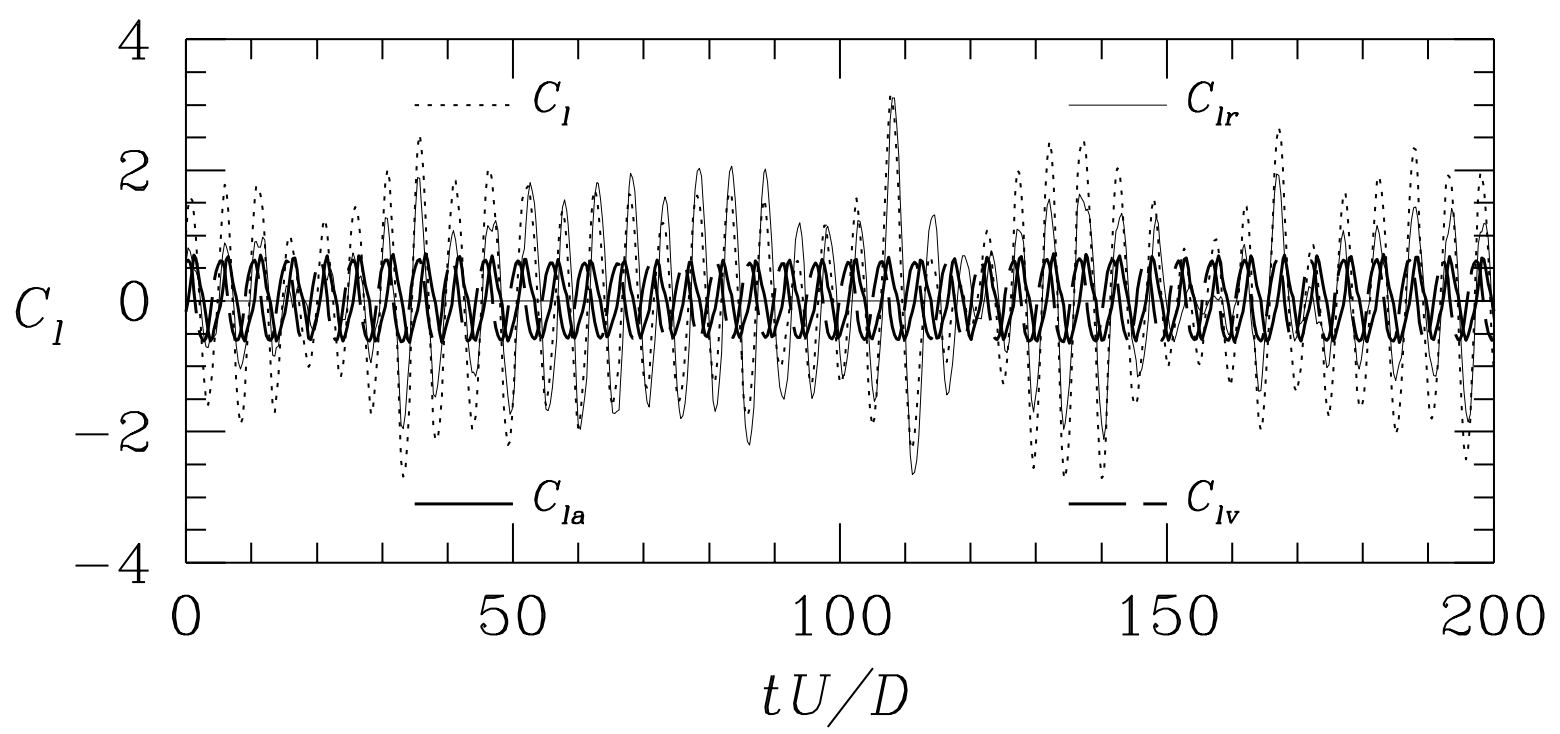

Figure 3: Example showing decomposition of lift force into parts correlated with cylinder acceleration and velocity, with uncorrelated residual. ....., $C_{l} ;-, C_{l r} ;-, C_{l a} ;--, C_{l v}$.

(a)

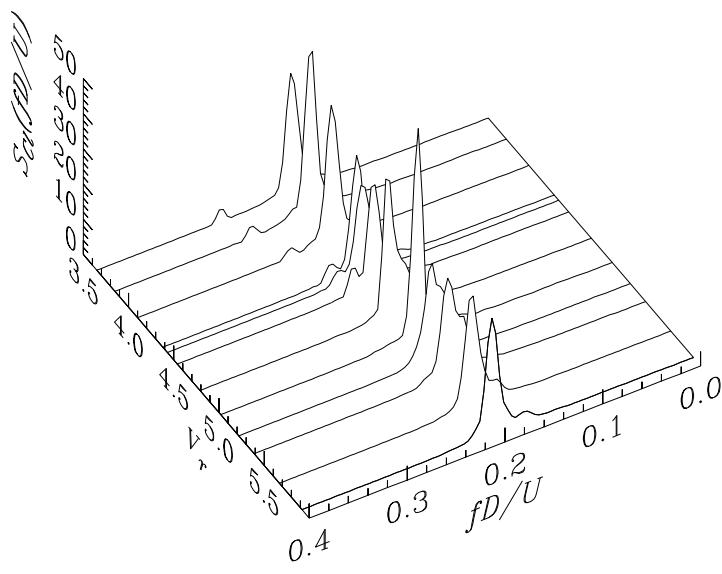

(b)

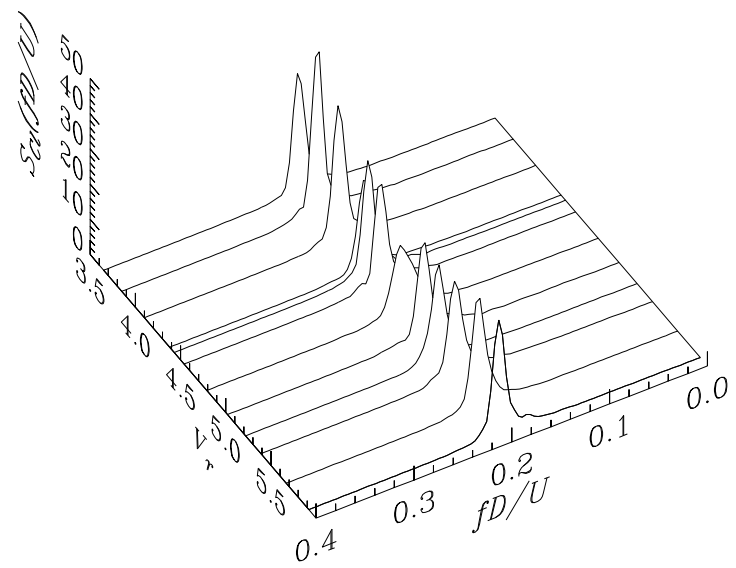

Figure 4: Variation with $V_{r}$ of lift force spectra for a nominal oscillation amplitude of $2.7 \% D$ in smooth flow: $(a)$ : total lift force, $(b)$ : residual lift force. 


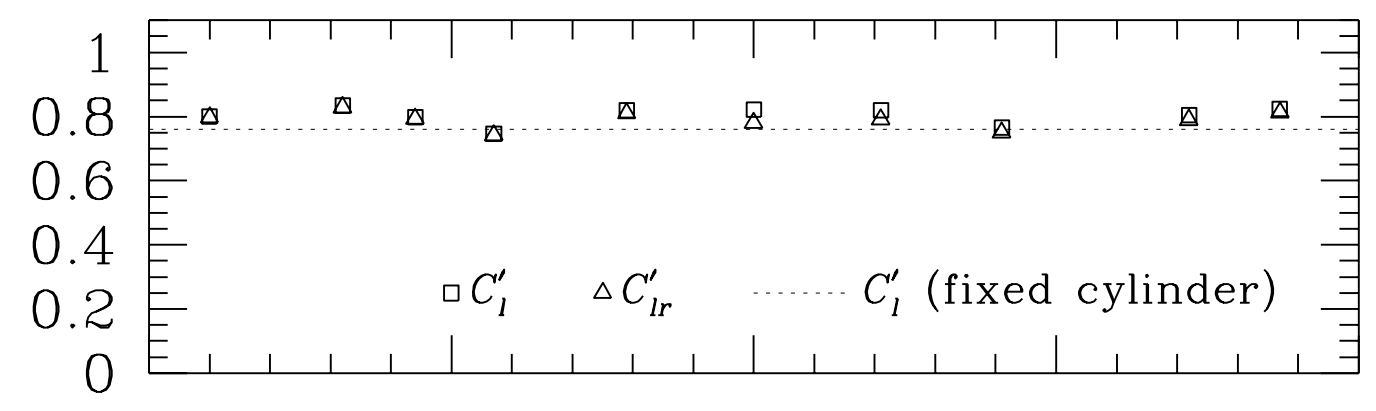

( a)

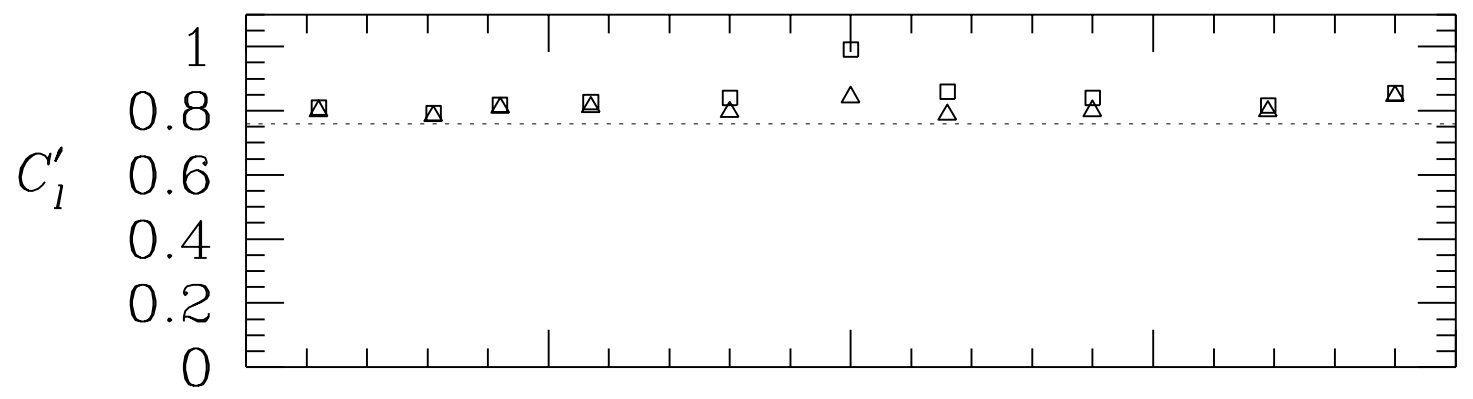

$(b)$

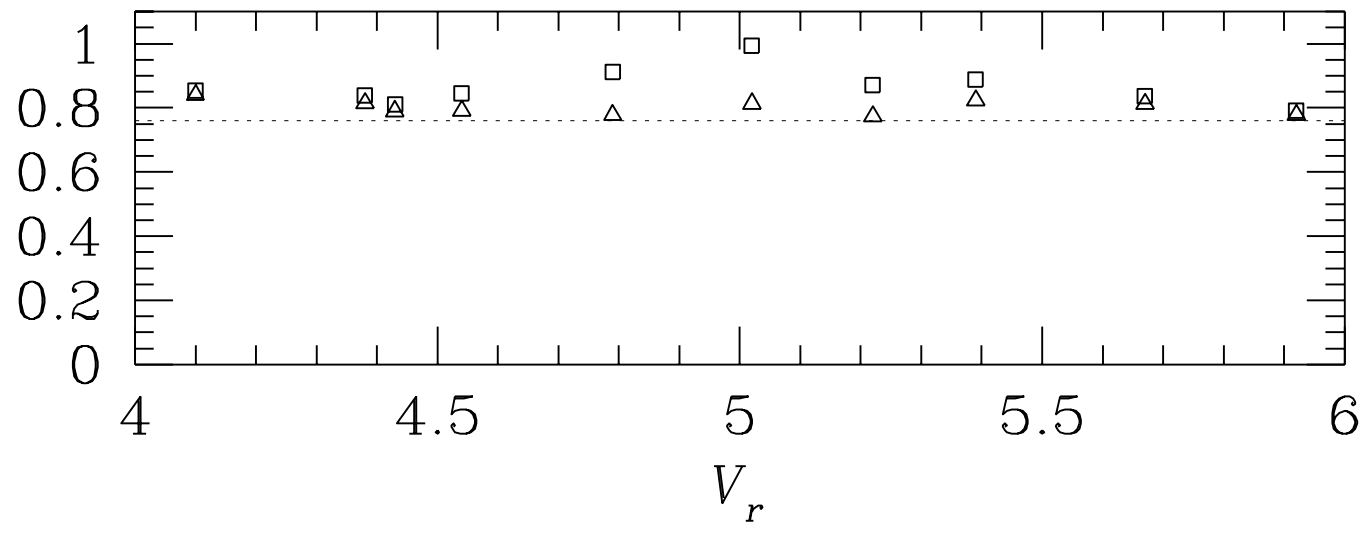

(c)

Figure 5: RMS values of total and residual lift in smooth flow. Lower dashed line in each graph indicates value of $C_{l}^{\prime}$ with cylinder held fixed at beginning of tests (0.76). Nominal oscillation amplitudes: (a), $\alpha=0.98 \%$; (b), $\alpha=1.9 \%$; (c), $\alpha=2.7 \% . \square, C_{l}^{\prime} ; \triangle, C_{l r}^{\prime} ; \cdots \cdots$, $C_{l}^{\prime}$ (fixed cylinder). 


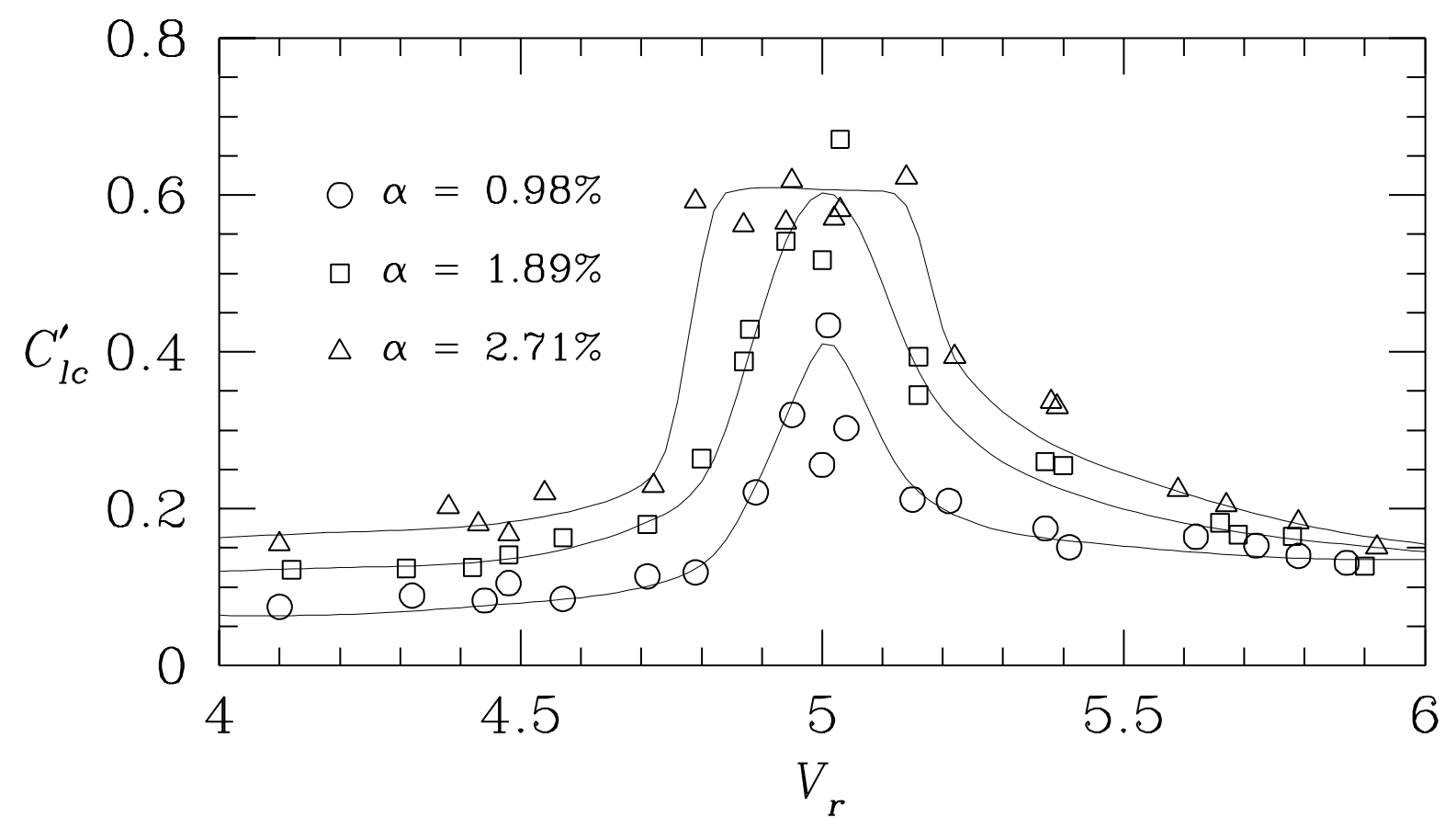

Figure 6: Values of the differential (motion-correlated) coefficients of lift $C_{l c}^{\prime}$ (eq. 10) in smooth flow as functions of reduced velocity and amplitude. $\circ, \alpha=0.98 \%$; $\square, \alpha=1.9 \%$; $\triangle, \alpha=2.7 \%$. 


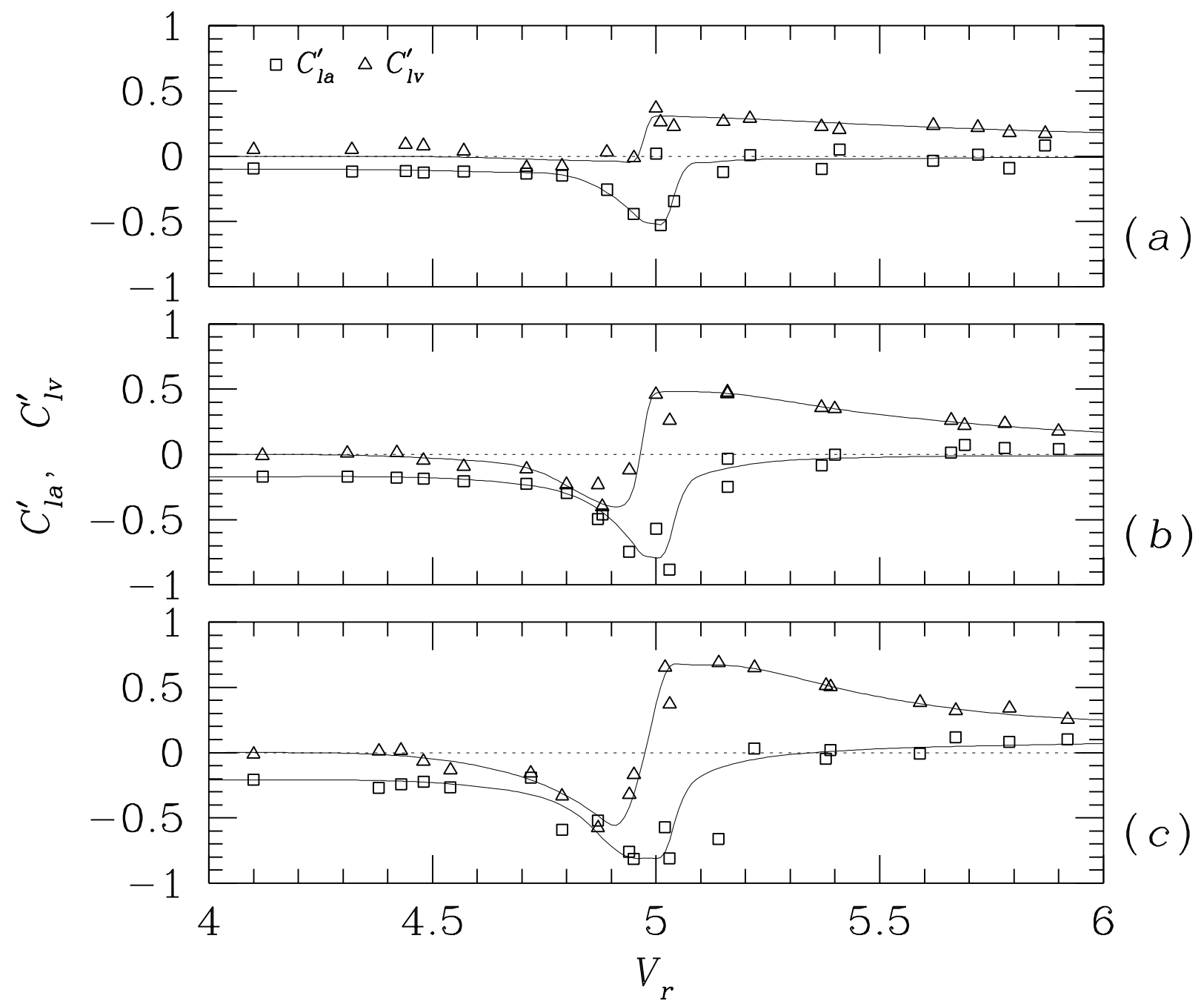

Figure 7: Coefficients of fluctuating lift correlated to cylinder acceleration $\left(C_{l a}^{\prime}\right)$ and velocity $\left(C_{l v}^{\prime}\right)$ in smooth flow as functions of $V_{r}$ at amplitudes of motion: (a): $\alpha=0.98 \%$, (b): $\alpha=1.9 \%,(c): \alpha=2.7 \% . \square, C_{l a}^{\prime} ; \triangle, C_{l v}^{\prime}$. 


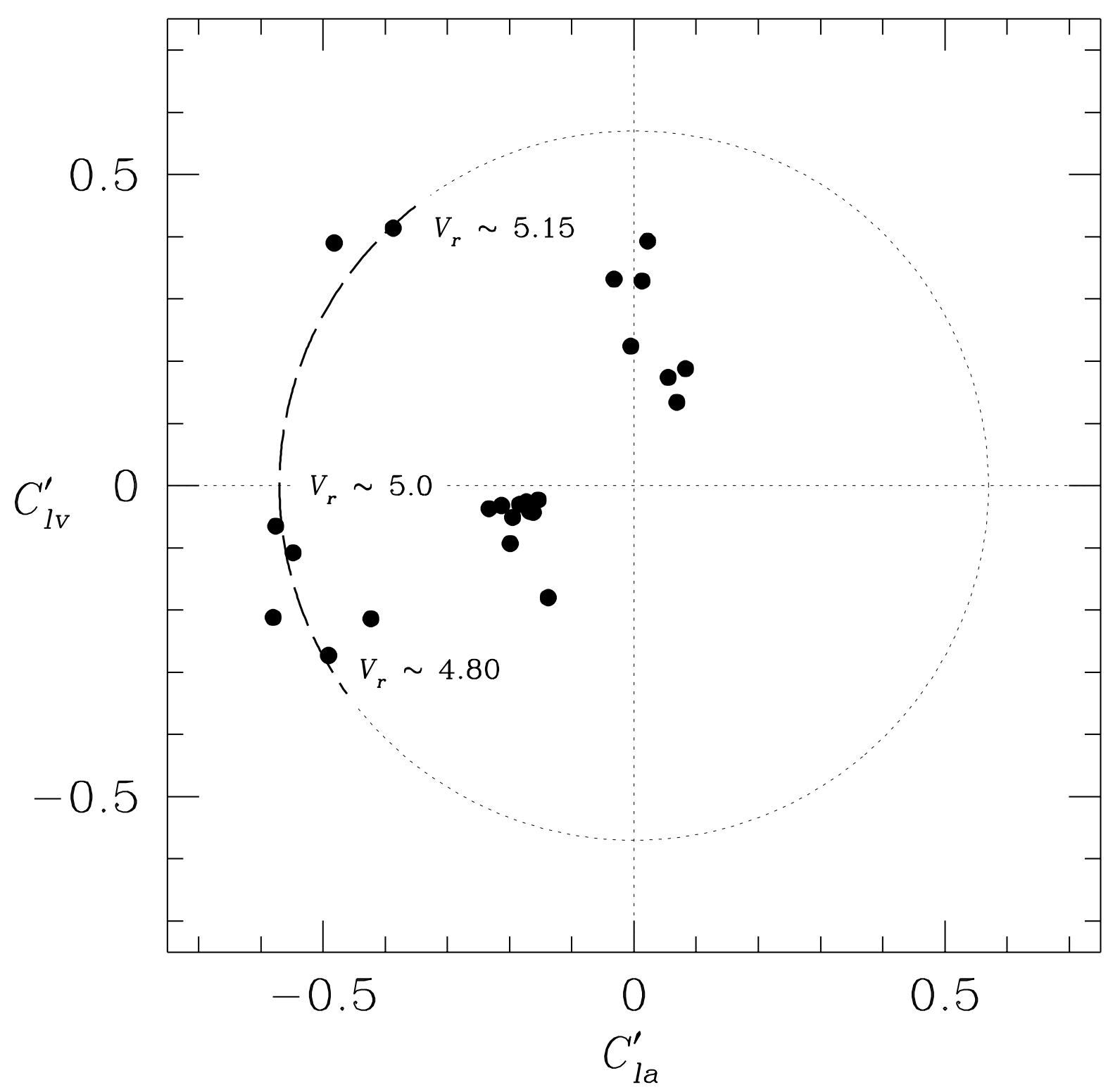

Figure 8: Coefficients of acceleration- and velocity-correlated lift $\left(C_{l a}^{\prime} \& C_{l v}^{\prime}\right)$ for $\alpha=2.7 \%$ in smooth flow, with $V_{r}$ as a parameter. 


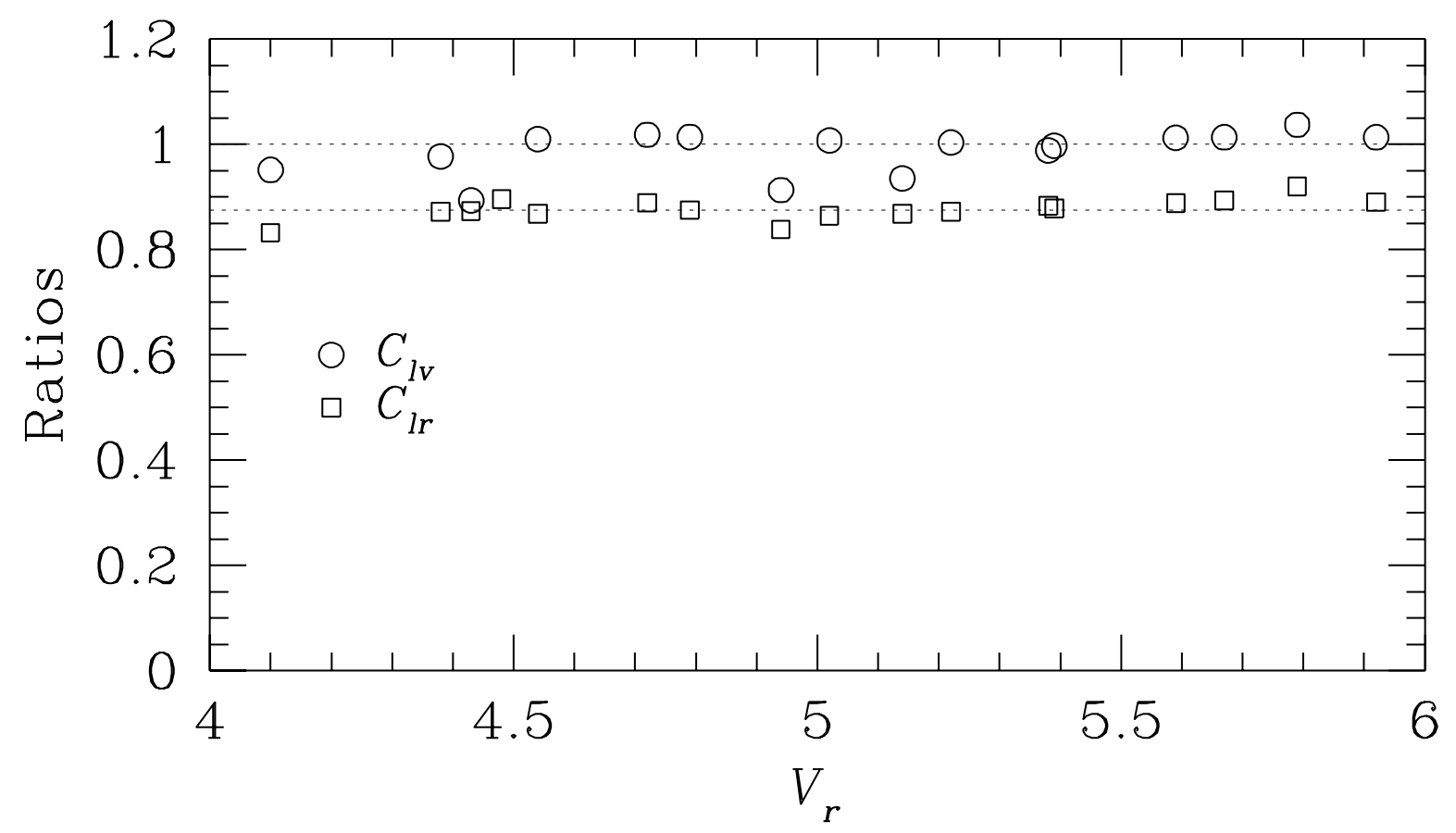

Figure 9: Comparison of ratio of value of $C_{l v}^{\prime}$ obtained from the spanwise-mean lift and cylinder velocity to the average value obtained from the six individual measurements to a similar ratio for the residual lift $C_{l r}^{\prime} . \alpha=2.7 \%$, smooth flow. $\circ, C_{l v}^{\prime} ; \square, C_{l r}^{\prime}$.
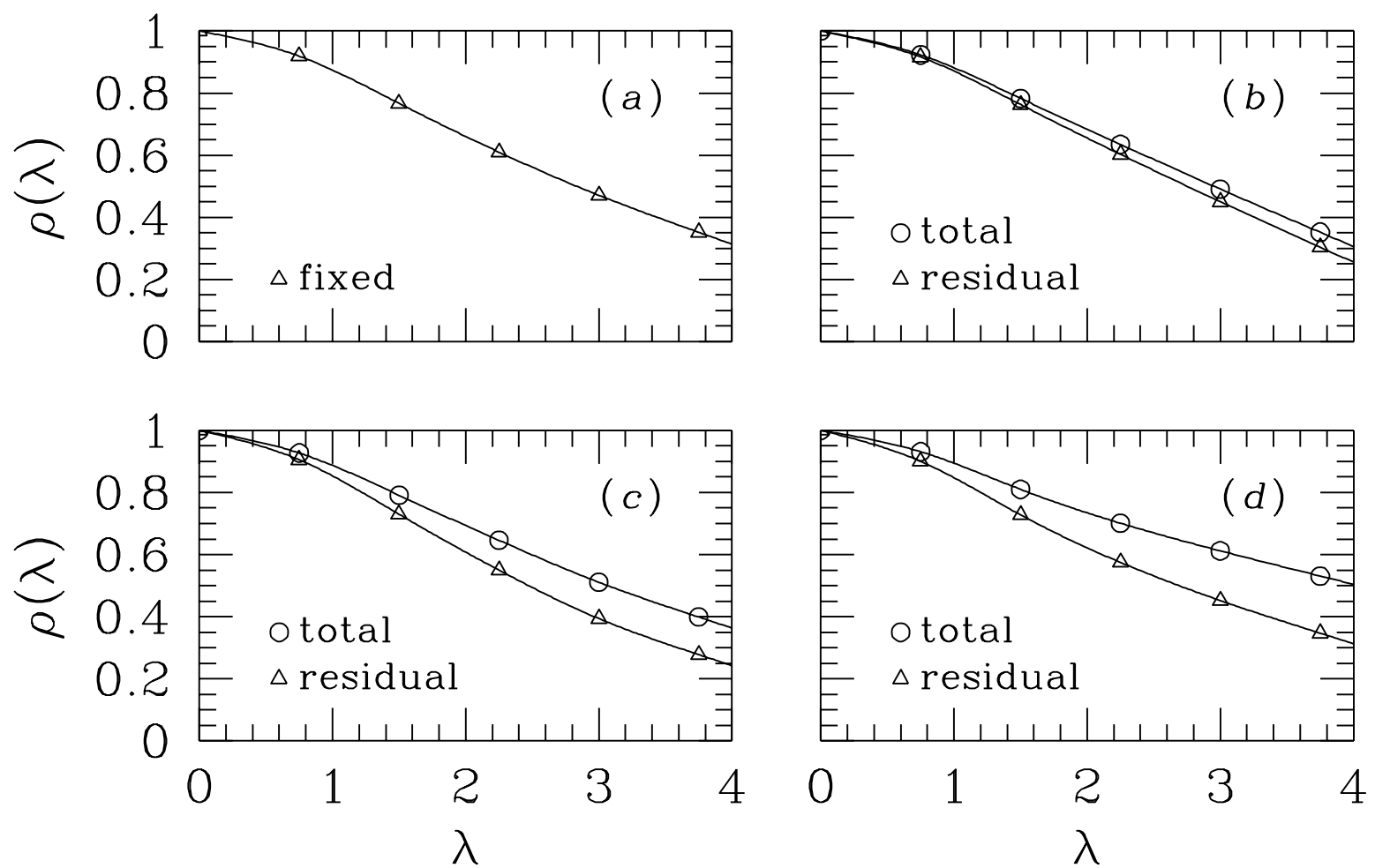

Figure 10: Effect of cylinder oscillation amplitude on spanwise correlation at $V_{r}=V_{r \mathrm{c}}$ in smooth flow; (a): fixed cylinder, (b): $\alpha=0.98 \%,(c): \alpha=1.9 \%,(d): \alpha=2.7 \%$. $\circ$, total; $\triangle$, residual. 


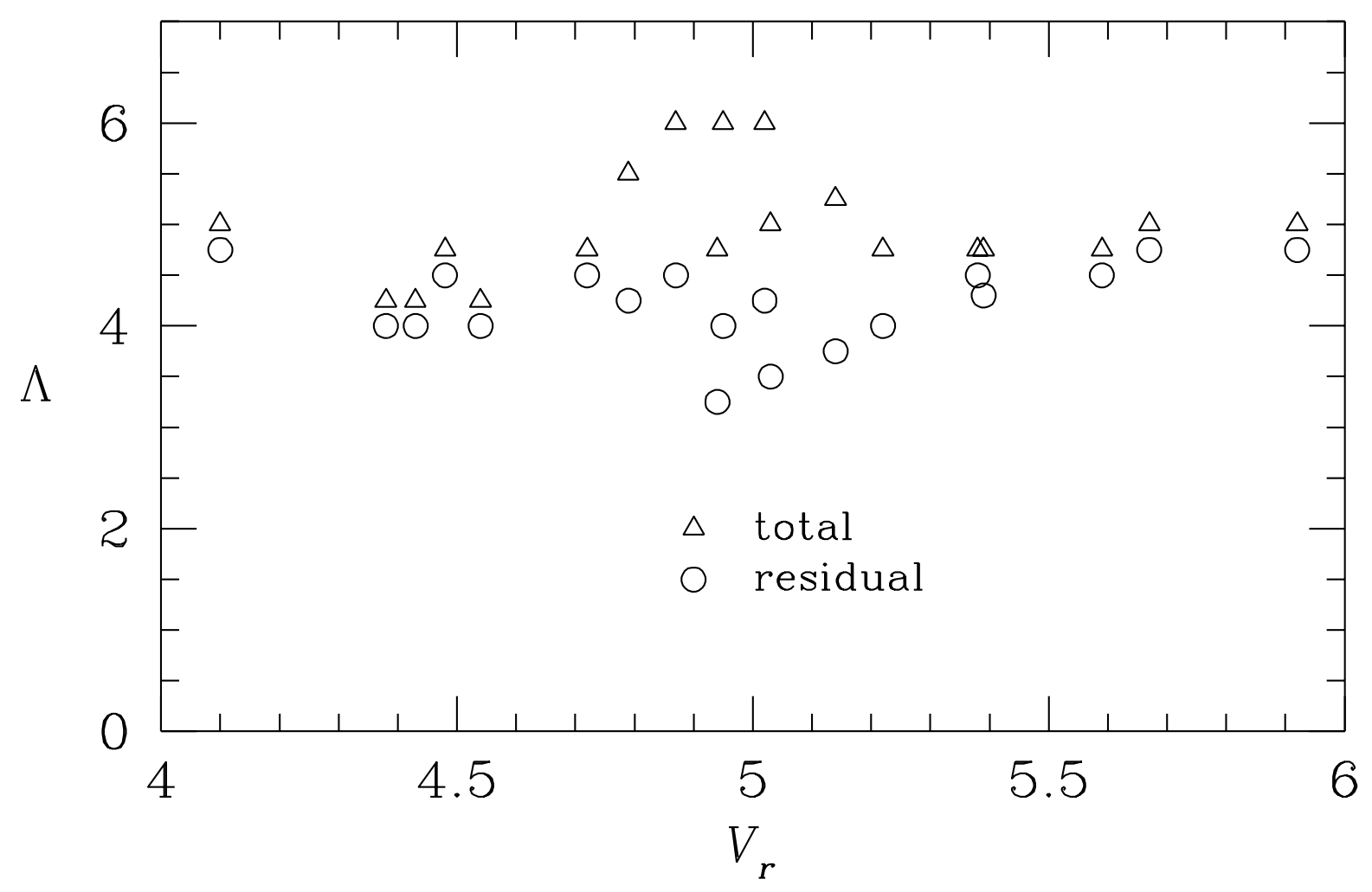

Figure 11: Spanwise correlation lengths $\Lambda$ for total and residual lift forces in smooth flow shown as functions of $V_{r}(\alpha=2.7 \%) . \triangle$, total; $\bigcirc$, residual. 


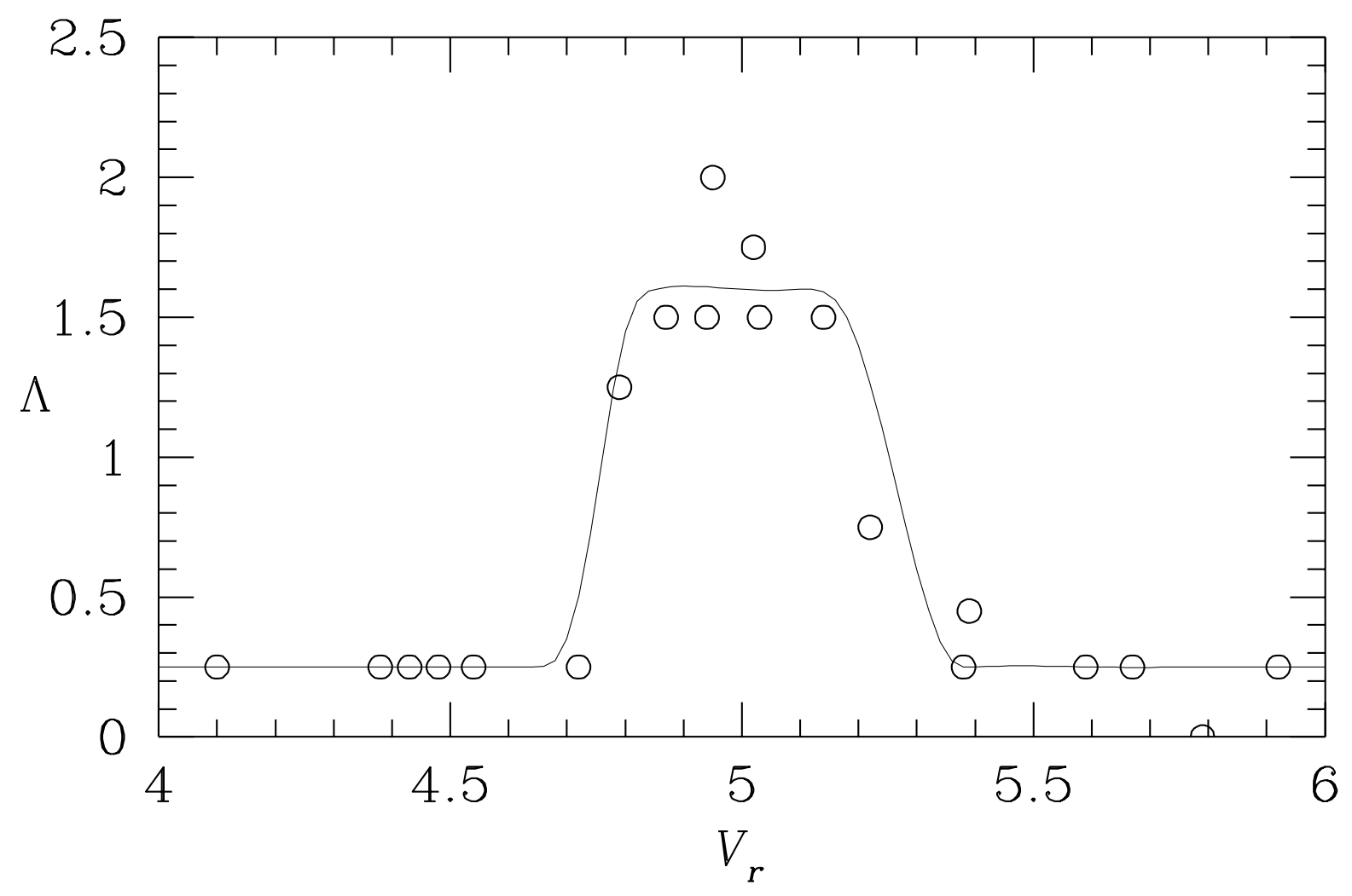

Figure 12: Difference between correlation lengths of total and residual lift forces as a function of $V_{r}(\alpha=2.7 \%)$ for smooth flow. 


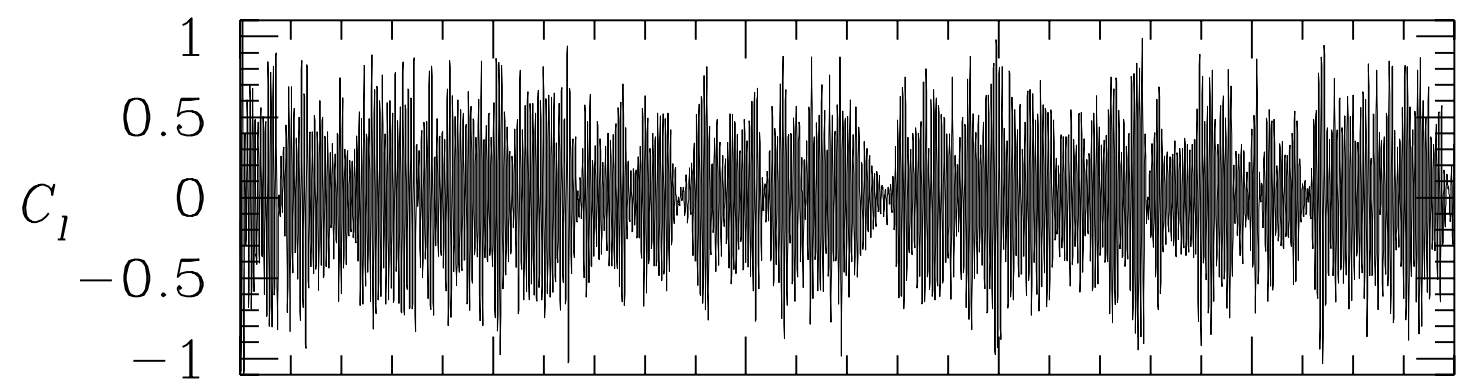

( a )

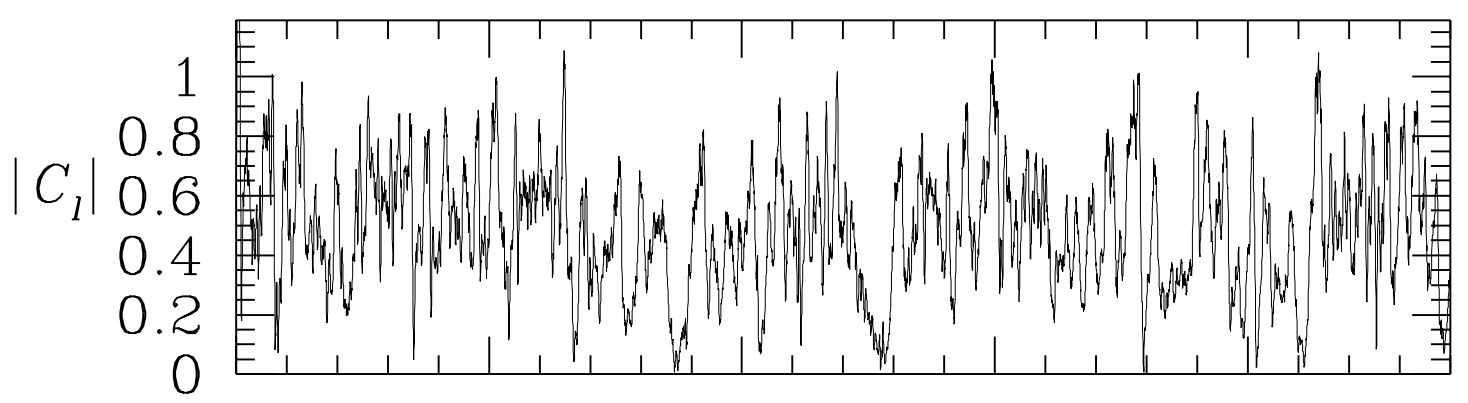

(b)

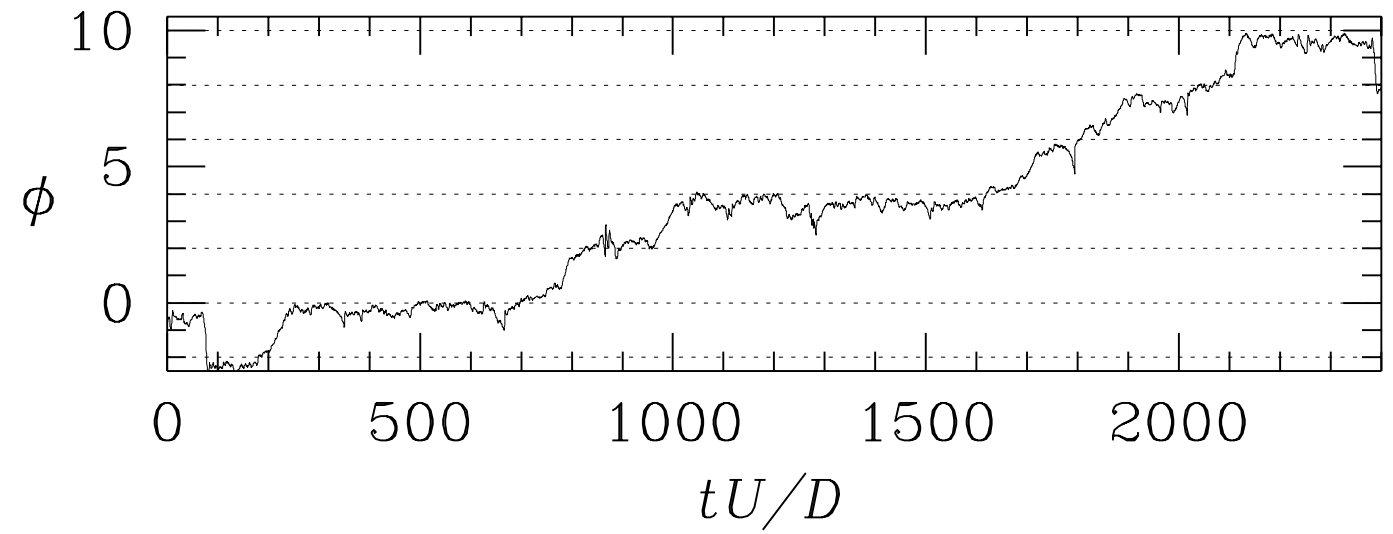

(c)

Figure 13: Lift coefficient (a), magnitude envelope $(b)$ and $\phi$, phase relative to cylinder velocity $(c)$ at $V_{r}=5.14, \alpha=2.7 \%$ in smooth flow. Phase angles are given as multiples of $\pi$. 


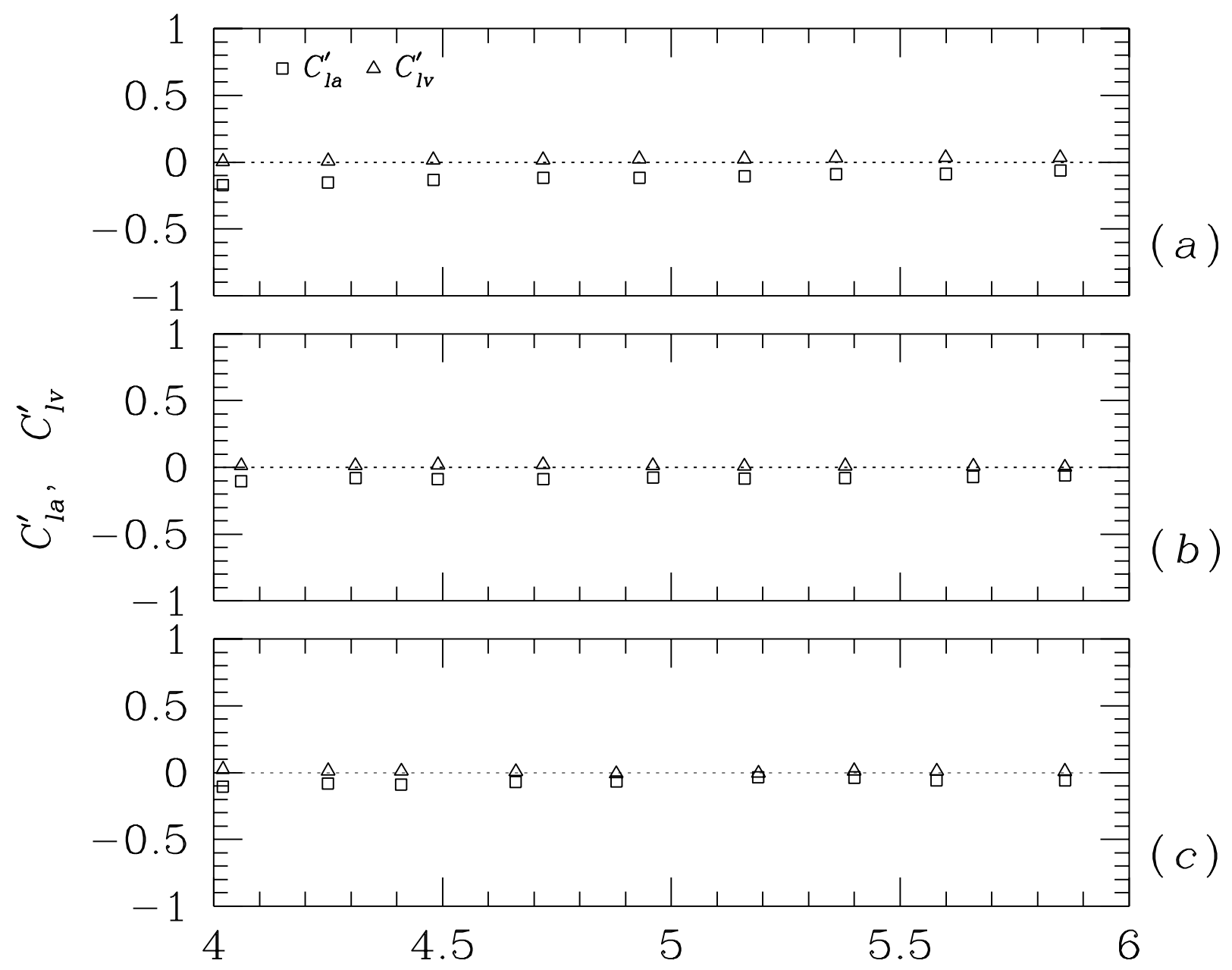

Figure 14: Coefficients of fluctuating lift correlated to cylinder acceleration $\left(C_{l a}^{\prime}\right)$ and velocity $\left(C_{l v}^{\prime}\right)$ as functions of $V_{r}$ for nominal motion amplitude of $3 \% D$ in turbulent flows. Turbulence intensities; (a): $I_{u}=4.2 \%,(b): I_{u}=9.6 \%,(c): I_{u}=18 \%$. $\square, C_{l a}^{\prime} ; \triangle, C_{l v}^{\prime}$. 